\title{
Some More New Properties of Consecutive Odd Numbers
}

\author{
Xingbo Wang ${ }^{1}$ \\ ${ }^{1}$ Department of Mechatronics, Foshan University, Foshan City, Guangdong Province, PRC. \\ Correspondence: Xingbo WANG, Department of Mechatronics, Foshan University, Foshan City, Guangdong Province, \\ PRC, 528000. E-mail: wxbmail@msn.com;xbwang@fosu.edu.cn
}

Received: August 14, 2017 Accepted: September 6, $2017 \quad$ Online Published: September 12, 2017

doi:10.5539/jmr.v9n5p61

URL: https://doi.org/10.5539/jmr.v9n5p61

\begin{abstract}
The article proves several new properties of consecutive odd integers. The proved properties reveal divisors' transition by subtracting two terms of an odd sequence, divisors' stationary with adding or subtracting an item to the terms and pseudo-symmetric distribution of a divisor's power in an odd sequence. The new properties are helpful for finding a divisor of an odd composite number in an odd sequence.
\end{abstract}

Keywords: odd integer, divisor, distribution, calculation

MSC 2000: 11A51,11A05

\section{Introduction}

Study of odd integers has been an important topic in number theory for several hundred years, as introduced in Dickson's book (Dickson, L. E., 1971). People have spent much time on studying the prime numbers, which are a special kind of odd integers, and obtained many excellent achievements as well as a lot of unsolved problems most of which are closely related with odd integers, as illustrated in Rosen's book (Rosen, K. H., 2011). Nowadays, the problem of factorizing a large odd number has still been a well-known difficult problem in the world, as Sarnaik S and Liu XX stated in their articles (Sarnaik, S., Gadekar, D., Gaikwad, U., 2014 ; Liu, X. X., Zou, X. X. \& Tan, J. L., 2014), and Kessler overviewed in his book (Kessler G C. 2016). It is indubitable that, study of odd integers in different perspectives is helpful for knowing both the prime numbers and the factorization of integers. Based on such a point of view, WANG made studies on odd integers by several different approaches and obtained many new properties (WANG Xingbo, 2014-2017). Following the previous studies, this article aims at discovering some more new properties of consecutive odd numbers and intends to provide a mathematical foundation in people's knowing the distributions of odd integers' divisors.

\section{Preliminaries}

This section introduces symbols, definitions and lemmas that are necessary in later sections.

\subsection{Symbols and Notations}

Throughout this paper, an odd sequence is defined to be a sequence of odd numbers, e.g., 13,15,19,23,31. An odd interval $[a, b]$ is a set of consecutive odd numbers that take $a$ as their lower bound and $b$ as their upper bound. For example, $[3,11]=\{3,5,7,9,11\}$. An odd interval $[a, b]$ is said to contain another odd interval $[c, d]$, denoted by $[c, d] \subset[a, b]$, if $a, b, c$ and $d$ satisfy one of the following three conditions

(1) $a \leq c$ and $d<b$;

(2) $a<c$ and $d \leq b$;

(3) $a<c$ and $d<b$.

Symbol $(a, b)$ denotes the greatest common divisor of integer $a$ and $b$. Symbol $v(m, n, p)$ denotes the number of $p$ 's multiples from integer $m$ to integer $n$. Symbol $\lfloor x\rfloor$ is to express $x$ 's floor function defined by $x-1<\lfloor x\rfloor \leq x$, where $x$ is a real number; and symbol $|x\rangle$ is to express $x$ 's odd floor function that is defined by

$$
|x\rangle=\left\{\begin{array}{l}
\lfloor x\rfloor, \text { when }\lfloor x\rfloor \text { is odd } \\
\lfloor x\rfloor-1, \text { when }\lfloor x\rfloor \text { is even }
\end{array}\right.
$$

\subsection{Lemmas}

Lemma 1 (See in Rosen's book,2011) Let $a, b, c$ and $r$ be integers such that $a=b c+r$; then $(a, b)=(b, r)$. If $m$ and $n$ are odd integers and $m>n$, then $(m-n, n)=(m, n)$ 
Lemma 2 (See in WANG Xingbo's, 2014\&2016) Let $p$ be a positive odd integer; then among $p$ consecutive positive odd integers there exists one and only one that can be divisible by $p$. Let $q$ be a positive odd number and $S$ be a finite set that is composed of consecutive odd integers; then $S$ needs at least $(n-1) q+1$ elements to have $n$ multiples of $q$.

Lemma 3. Let $m, n$ and $p$ be positive integers such that $1<p<m<n$; then number of $p$ 's multiples from $m$ to $n$ is calculated by

$$
v(m, n, p)=\left\{\begin{array}{l}
\left\lfloor\frac{n}{p}\right\rfloor-\left\lfloor\frac{m}{p}\right\rfloor, p \nmid m \\
\left\lfloor\frac{n}{p}\right\rfloor-\left\lfloor\frac{m}{p}\right\rfloor+1, p \mid m
\end{array}\right.
$$

Proof. It is known that, there are $\left\lfloor\frac{m}{p}\right\rfloor p$ 's multiples from 1 to $m$. Let $r_{m}=m-p\left\lfloor\frac{m}{p}\right\rfloor$ and group the all the integers from 1 to $m$ by a unit that contains $p$ consecutive integers. It can see that there are $\left\lfloor\frac{m}{p}\right\rfloor$ complete units, each of which contains $p$ consecutive integers, and an incomplete unit that contains only $r_{m}$ consecutive integers, as shown in figure 1 .

$$
\underbrace{1,2,3, \ldots, p}_{p}, \underbrace{p+1, \ldots, 2 p}_{p} \ldots, \underbrace{\left(\left\lfloor\frac{m}{p}\right\rfloor-1\right) p+1 \ldots,\left\lfloor\frac{m}{p}\right\rfloor p}_{r_{m}}, \underbrace{\left\lfloor\frac{m}{p}\right\rfloor p+1 \ldots, m}
$$

Figure 1. Grouped $m$ integers

Now group all the integers from 1 to $n$ in the same way, as shown in figure 2 .

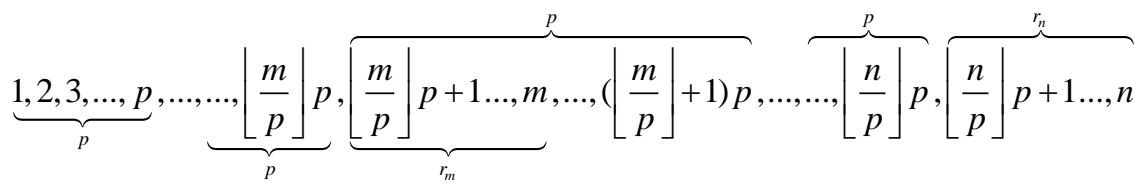

Fig 2 Grouped $n$ integers

Then it can see that, if $p \nmid m$, the integers $\left(\left\lfloor\frac{m}{p}\right\rfloor+1\right) p, \ldots,\left\lfloor\frac{n}{p}\right\rfloor p$ are $p$ 's multiples. The number of the multiples is $\left\lfloor\frac{n}{p}\right\rfloor-\left(\left\lfloor\frac{m}{p}\right\rfloor+1\right)+1=\left\lfloor\frac{n}{p}\right\rfloor-\left\lfloor\frac{m}{p}\right\rfloor$. If $p \mid m$, the integers, $\left.\left\lfloor\frac{m}{p}\right\rfloor p, \quad\left\lfloor\frac{m}{p}\right\rfloor+1\right) p, \ldots,\left\lfloor\frac{n}{p}\right\rfloor p \quad$ are $p$ 's multiples which include $\left\lfloor\frac{n}{p}\right\rfloor-\left\lfloor\frac{m}{p}\right\rfloor+1$ integers in all.

\section{Theorems and Proofs}

Theorem 1. Let $n$ be a positive integer and $S=\left\{s_{1}, s_{2}, \ldots, s_{n}\right\}$ be a sequence that consists in $n$ consecutive odd integers; if odd number $p$ has one and only one multiple in $S$, then $p \geq\left\lfloor\frac{n}{2}\right\rfloor$.

Proof. Use proof by contradiction. Assume $\quad p<\left\lfloor\frac{n}{2}\right\rfloor$, then it yields $\quad p \leq\left\lfloor\frac{n}{2}\right\rfloor-1$. Since $n=\lfloor n\rfloor$, it knows

$$
\left.n-p \geq\lfloor n\rfloor-\left(\frac{n}{2}\right\rfloor-1\right)=\left\lfloor 2 \cdot \frac{n}{2}\right\rfloor-\left\lfloor\frac{n}{2}\right\rfloor+1 \geq 2\left\lfloor\frac{n}{2}\right\rfloor-\left\lfloor\frac{n}{2}\right\rfloor+1=\left\lfloor\frac{n}{2}\right\rfloor+1
$$

This indicates by Lemma 2 and Lemma 3 that there are at least 2 terms that are $p$ 's multiples in $S$, which is contradictory to the condition that $S$ contains exact one $p$ 's term. 
Theorem 2. Let $p$ be an odd number and $n$ be a positive integer with $n>p$. Suppose $a_{1}, a_{2}, \ldots, a_{n}$ are $n$ consecutive odd numbers and $S=\left\{a_{j}-a_{i} \mid 1 \leq i<j \leq n\right\}$; then there are $v(p, n)=\alpha n-\frac{\alpha(\alpha+1)}{2} p p$ 's multiples in $S$, where $\alpha=\left\lfloor\frac{n}{p}\right\rfloor$.

Proof. Take arbitrary two numbers $a_{i}$ and $a_{j}$, where $1 \leq i<j \leq n$. Since $a_{i}$ and $a_{j}$ are odd numbers, $a_{j}-a_{i}$ is surely an even number. Therefore, $p$ 's multiples that are produced by $a_{j}-a_{i}$ must be $2 p, 4 p, 6 p, \ldots$. Without loss of generality, suppose $a_{1}=2 s+1$, where $s$ is a positive integer; then $a_{i}=2(s+i-1)+1$ and $a_{j}=2(s+j-1)+1$. This yields

$$
a_{j}-a_{i}=2(j-i)
$$

and when $j=i+k$ it results in

Consequently it discovers the following facts.

$$
a_{i+k}-a_{i}=2 k
$$

(1) There are $n-1$ pairs of $a_{i}$ and $a_{j}$ such that $a_{j}-a_{i}=2$; the $n-1$ numbers are $a_{i+1}-a_{i}=2$ when $i=1,2, \ldots, n-1$.

(2) There are $n-2$ pairs of $a_{i}$ and $a_{j}$ such that $a_{j}-a_{i}=4$; the $n-2$ numbers are $a_{i+2}-a_{i}=4$.

(3) There are $n-k$ pairs of $a_{i}$ and $a_{j}$ such that $a_{j+k}-a_{i}=2 k$; the $n-k$ numbers are $a_{i+k}-a_{i}=2 k$.

Consequently, it knows that there are totally $n-p$ numbers that are of the form $2 p$ which is produced by $a_{i+p}-a_{i}=2 p$, there are totally $n-2 p$ numbers that are of the form $4 p$ which is produced by $a_{i+2 p}-a_{i}=4 p$, and when $n>\alpha p$ there are totally $n-\alpha p$ numbers that are of the form $2 \alpha p$ which is produced by $a_{i+2 \alpha p}-a_{i}=2 \alpha p$. As a result, the total number $v(p, n)$ of $p$ 's multiples in $S=\left\{a_{j}-a_{i} \mid 1 \leq i<j \leq n\right\}$ is given by

$$
v(p, n)=(n-p)+(n-2 p)+\ldots+(n-\alpha p)=\alpha n-\frac{\alpha(\alpha+1)}{2} p
$$

where $\alpha=\left\lfloor\frac{n}{p}\right\rfloor$.

Example 1. Let $p=3$ and $S=\{3,5,7,9,11,13\}$; then $n=6, \alpha=2$ and

$$
v(3,6)=2 \times 6-\frac{2 \times 3}{2} \times 3=3
$$

which says there are three 3 's multiples that are produced by subtracting arbitrary two elements in $S$. In fact, it can see that the 3 multiples are $9-3=6,11-5=6$ and $13-7=6$.

Theorem 3. Let $p$ be an odd integer and $n$ be a positive integer with $n>p$. Suppose $S=\left\{a_{1}, a_{2}, \ldots, a_{n}\right\}$ is composed of $n$ consecutive odd integers with $a_{1}>p$; if $S^{*}=\left\{a_{i}-p \mid 1 \leq i \leq n\right\}$, then the number $v$ of $p$ 's multiples in $S^{*}$ is estimated by

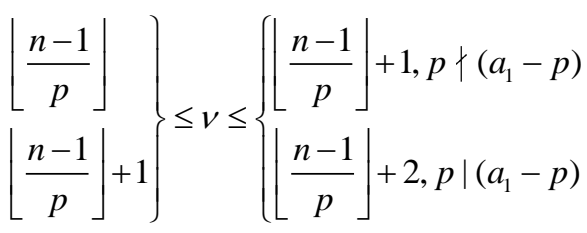

Proof. Since $p$ and $a_{1}$ are odd integers, let $a_{1}-p=2 s$; then the set $S^{*}$ can be equivalently rewritten by

$$
S^{*}=\{2(s+i) \mid 0 \leq i \leq n-1\}
$$

Let

$$
\tilde{S}=\{s+i \mid 0 \leq i \leq n-1\}
$$


then terms in $\tilde{S}$ are one-to-one mapping to the terms in $S^{*}$ and $\tilde{S}$ contains $n$ consecutive integers. By Lemma 3, the number of $p$ 's multiples in $\tilde{S}$ is calculated by

$$
v\left(a_{1}-p, a_{1}-p+n-1, p\right)=\left\{\begin{array}{l}
\left\lfloor\frac{a_{1}-p+n-1}{p}\right\rfloor-\left\lfloor\frac{a_{1}-p}{p}\right\rfloor, p \nmid\left(a_{1}-p\right) \\
\left\lfloor\frac{a_{1}-p+n-1}{p}\right\rfloor-\left\lfloor\frac{a_{1}-p}{p}\right\rfloor+1, p \mid\left(a_{1}-p\right)
\end{array}\right.
$$

Since $\left\lfloor\frac{n-1}{p}\right\rfloor \leq\left\lfloor\frac{a_{1}-p+n-1}{p}\right\rfloor-\left\lfloor\frac{a_{1}-p}{p}\right\rfloor \leq\left\lfloor\frac{n-1}{p}\right\rfloor+1$, it yields

$$
\left.\begin{array}{l}
\left\lfloor\frac{n-1}{p}\right\rfloor \\
\left\lfloor\frac{n-1}{p}\right\rfloor+1
\end{array}\right\} \leq v\left(a_{1}-p, a_{1}-p+n-1, p\right) \leq\left\{\begin{array}{l}
\left\lfloor\frac{n-1}{p}\right\rfloor+1, p \nmid\left(a_{1}-p\right) \\
\left\lfloor\frac{n-1}{p}\right\rfloor+2, p \mid\left(a_{1}-p\right)
\end{array}\right.
$$

Corollary 1. Let $p$ be an odd integer and $n$ be a positive integer with $n \leq p$. Suppose $S=\left\{a_{1}, a_{2}, \ldots, a_{n}\right\}$ is composed of $n$ consecutive odd integers with $a_{1}>p$ and $S^{*}=\left\{a_{i}-p \mid 1 \leq i \leq n\right\}$; then $S$ contains at most one $p$ 's multiple and

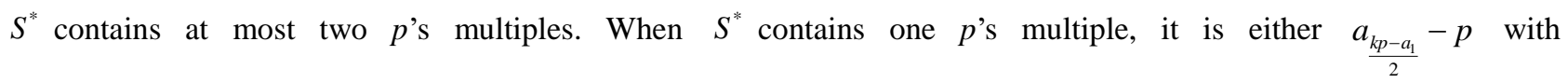
$\left\lfloor\frac{a_{1}+2}{p}\right\rfloor+1 \leq k \leq\left\lfloor\frac{2 n+a_{1}}{p}\right\rfloor$ or $\quad a_{\frac{(k+1) p-a_{1}}{2}}-p$ with $\left\lfloor\frac{a_{1}+2}{p}\right\rfloor \leq k \leq\left\lfloor\frac{2 n+a_{1}}{p}\right\rfloor-1$; when $S^{*}$ contains two $p$ 's multiples, $S=\{(2 k+1) p,(2 k+1) p+2, \ldots,(2 k+3) p\}$ and $S^{*}=\{2 k p, 2 k p+2, \ldots, 2(k+1) p\}$.

Proof. By Lemma 2, if $S$ contains more than one $p$ 's multiples, then $n \geq p+1$. That is contradictory to $n \leq p$. Hence $S$ contains at most one $p$ 's multiples.

Now consider $p$ 's multiples in $S^{*}$. Note that $n \leq p$ leads to $\left\lfloor\frac{n-1}{p}\right\rfloor=0$. By Theorem 2, there are two cases, $p \nmid\left(a_{1}-p\right)$ and $p \mid\left(a_{1}-p\right)$, to be investigated. First consider the case $p \nmid\left(a_{1}-p\right)$. This time $S^{*}$ contains at most one $p$ 's multiples by Theorem 2. Actually, it can show that $\frac{a_{k p-a_{1}}}{2}-p$ is $p$ 's multiple when $\left\lfloor\frac{a_{1}+2}{p}\right\rfloor+1 \leq k \leq\left\lfloor\frac{2 n+a_{1}}{p}\right\rfloor$, and $\quad \frac{a_{(k+1) p-a_{1}}}{2}-p$ is $p$ 's multiple when $\left\lfloor\frac{a_{1}+2}{p}\right\rfloor \leq k \leq\left\lfloor\frac{2 n+a_{1}}{p}\right\rfloor-1$.

Direct calculations show

$$
a_{\frac{k p-a_{1}}{2}}-p=a_{1}+2 \cdot \frac{k p-a_{1}}{2}-p=(k-1) p
$$

and

which leads to

$$
a_{\frac{(k+1) p-a_{1}}{2}}-p=a_{1}+2 \cdot \frac{(k+1) p-a_{1}}{2}-p=k p
$$

$$
\left(a_{\frac{(k+1) p-a_{1}}{2}}-p\right)-\left(a_{\frac{k p-a_{1}}{2}}-p\right)=p
$$




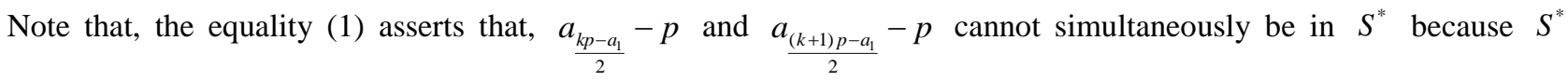

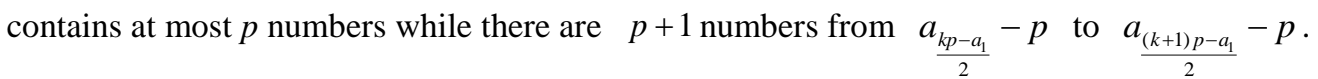

Now considering $\left\lfloor\frac{a_{1}+2}{p}\right\rfloor+1 \leq k \leq\left\lfloor\frac{2 n+a_{1}}{p}\right\rfloor$ yields $\quad \frac{a_{2}}{p}<k \leq \frac{2 n+a_{1}}{p}$, namely $1<\frac{k p-a_{1}}{2} \leq n \quad$ and $\left\lfloor\frac{a_{1}+2}{p}\right\rfloor \leq k \leq\left\lfloor\frac{2 n+a_{1}}{p}\right\rfloor-1$ yields $1<\frac{(k+1) p-a_{1}}{2} \leq n$, it knows that the first assertion of the corollary is sure to hold.

Now it turns to the case $p \mid\left(a_{1}-p\right)$. This time $S^{*}$ might contain at least 1 and at most $2 p$ 's multiples by Theorem 2 . In fact, $p \mid\left(a_{1}-p\right)$ means $a_{1}=p(2 k+1)$ for some integer $k \geq 1$ since $a_{1}$ and $p$ are odd; then $a_{i}-p=a_{1}+2 i-p=2 k p+2 i$; when $i=p, a_{i}-p$ is a multiple of $p$. Consequently, $n=p$ is the solution that $S^{*}$ contains 2 multiples of $p$. The solution is

$$
S=\{(2 k+1) p,(2 k+1) p+2, \ldots,(2 k+3) p\} \text { and } S^{*}=\{2 k p, 2 k p+2, \ldots, 2(k+1) p\}
$$

Corollary 2. Let $p$ be an odd integer and $n$ be a positive integer with $n \leq p$. Suppose $S=\left\{a_{1}, a_{2}, \ldots, a_{n}\right\}$ is composed of $n$ consecutive odd integers with $a_{1}>p$ and it contains exact one $p$ 's multiple. If $S^{*}=\left\{a_{i}-p \mid 1 \leq i \leq n\right\}$, then $S$ contains exact one $p$ 's multiple if $p \nmid a_{1}$ or $p \mid a_{1}$ together with $n<p ; S$ contains exact two $p$ 's multiples if $p \mid a_{1}$ and $n=p$.

Proof. (Omitted)

Theorem 4. Given $p$ is an odd integer bigger than $1, n$ is a positive integer with $n \leq p$ and $S=\left\{a_{1}, a_{2}, \ldots, a_{n}\right\}$ is composed of $n$ consecutive odd integers with $a_{1} \geq e p$ for an even integer $e \geq 2$; let $S^{*}=\left\{a_{i}-e p \mid 1 \leq i \leq n\right\}$; if $S$ contains exact one $p$ 's multiple $a_{m}$, then $a_{m}$-ep is the unique $p$ 's multiple in $S^{*}$.

Proof. Obviously, $S^{*}$ contains $n$ consecutive odd numbers and $a_{m}-e p$ is a $p$ 's multiple since $a_{m}=p s$ for some odd integer $s>1$. If $S^{*}$ contains some other $p$ 's multiples, then by Lemma 2 it needs at least $p+1$ numbers in $S^{*}$. This is contradictory to $n \leq p$. Hence the theorem holds.

Corollary 3. Given $p$ is an odd integer, $n$ is a positive integer with $n \leq p, S=\left\{a_{1}, a_{2}, \ldots, a_{n}\right\}$ is composed of $n$ consecutive odd integers with $2 \alpha p<a_{1} \leq 2(\alpha+1) p$ or for an integer $\alpha \geq 1$ and $S^{*}=\left\{s_{i} \mid s_{i}=a_{i}-2 \alpha p, 1 \leq i \leq n\right\}$; suppose $S$ contains only one $p$ 's multiple; then $S^{*}$ also contains only one $p$ 's multiple which is either $s_{\frac{(2 k+1) p-a_{1}}{2}}$ with $2 k p<a_{1} \leq(2 k+1) p$ or $s_{\frac{(2 k+3) p-a_{1}}{2}}$ with $(2 k+1) p<a_{1} \leq(2 k+3) p$. And thus the sole $p$ 's multiple in $S$ is either

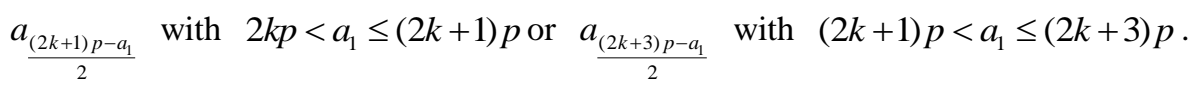

Proof. Let $a_{1}=2 \alpha p+r$; then $r$ is odd and $1 \leq r \leq 2 p-1$. Obviously $S^{*}$ can be rewritten by

$$
S^{*}=\{r, r+2, \ldots, r+2 s, \ldots, r+2(n-1)\}
$$

with $r$ and $r+2(n-1)$ being respectively the smallest term and the biggest term.

Since $1 \leq n \leq p$, it holds $r+2(n-1) \leq 4 p-3$. Hence the suspicious $p$ 's multiples in $S^{*}$ are either $r+(p-r)$ with $r \leq p$ or $r+(3 p-r)$ with $p<r \leq 3 p$. Direct calculations shows

$$
\begin{gathered}
\frac{s_{(2 k+1) p-a_{1}}}{2}=r+(2 k+1) p-a_{1}=p \\
s_{\frac{(2 k+3) p-a_{1}}{2}}=r+(2 k+3) p-a_{1}=3 p
\end{gathered}
$$




$$
a_{\frac{(2 k+1) p-a_{1}}{2}}=a_{1}+(2 k+1) p-a_{1}=(2 k+1) p
$$

and

$$
a_{\frac{(2 k+3) p-a_{1}}{2}}=(2 k+3) p
$$

Example 2. Let $p=127, S=\{1297,1299, \ldots, 1395,1397,1399, \ldots, 1497\}$; then $a_{1}=1297, \alpha=5$ and $r=27$. Since $127 \times 10<1297<127 \times 11$, it yields

$$
\frac{s_{1397-1297}^{2}}{2}=s_{50}=27+2 \times 50=127
$$

Theorem 5. Let $p$ be an odd integer, $n$ be a positive integer, $S=\left\{a_{1}, a_{2}, \ldots, a_{n}\right\}$ be composed of $n$ consecutive odd integers with $a_{1}>\alpha p$ for an integer $\alpha \geq 1$ and $S^{*}=\left\{s_{i} \mid s_{i}=a_{i}-\alpha p, 1 \leq i \leq n\right\}$; then $\left(p, a_{i}\right)=\left(p, s_{i}\right)$ for $1 \leq i \leq n$.

Proof. Rewrite $s_{i}=a_{i}-\alpha p$ by

$$
a_{i}=\alpha p+s_{i}, 1 \leq i \leq n
$$

Then by Lemma 1it yields

$$
\left(a_{i}, p\right)=\left(p, s_{i}\right), 1 \leq i \leq n
$$

Theorem 6. Let $a$ and $p$ be two odd integers with $p \mid a$. Suppose $p=2^{\alpha} s^{\beta}+1$ (or $p=2^{\alpha} s^{\beta}-1$ ), where $s>1$ is odd, $\alpha$ and $\beta$ are positive integers with $\alpha \geq 1$ and $\beta>0$; then there must exist an odd integer $b$ and an odd integer $c$ such that

(1) $b<a<c$ and $s^{\beta} \mid(b, c)$;

(2) there are at most $s^{\beta}+1$ consecutive odd integers from $b$ to $a$ or from $c$ to $a$.

Proof. First prove the case $p=2^{\alpha} s^{\beta}+1$. Let $\ldots, a-2 k, \ldots, a-2, a, a+2, \ldots, a+2 k, \ldots$ be consecutive odd integers; then by Lemma 2 , it knows that, among $s^{\beta}$ consecutive odd integers next to $a$ 's left there must be a $b$ such that $s^{\beta} \mid b$. Since b is among the $s^{\beta} a$ 's consecutive odd neighboring integers, it knows there are at most $s^{\beta}+1$ consecutive odd numbers from $b$ to $a$. Similarly, it knows that, among $s^{\beta}$ consecutive odd integers next to $a$ 's right there must exist a $c$ such that $s^{\beta} \mid c$ and there are at most $s^{\beta}+1$ consecutive odd numbers either from $c$ to $a$ or from $b$ to $a$. The case $p=2^{\alpha} s^{\beta}-1$ can be proved in the same way.

Corollary 4. Let $a$ and $p$ be two odd numbers and $p \mid a$. Suppose $p=2^{\alpha} s^{\beta}+1$ (or $p=2^{\alpha} s^{\beta}-1$ ), where $s>1$ is odd, $\alpha$ and $\beta$ are positive integers with $\alpha \geq 1$ and $\beta>0$; then among $s^{\beta}$ consecutive odd numbers next to $a$ 's left there are odd numbers $b_{i}$ such that $s^{i} \mid b_{i}$, where $i=\beta, \beta-1, \ldots, 2,1$, and among $s^{\beta}$ consecutive odd numbers next to $a$ 's right there are $c_{j}$ such that $s^{j} \mid c_{j}$, where $j=1,2, \ldots, \beta$.

Proof.(Omitted)

Corollary 5. Let $a$ and $p$ be two odd integers and $p \mid a$. Suppose $p=2^{\alpha} s^{\beta} t^{\sigma}+1$ (or $p=2^{\alpha} s^{\beta} t^{\sigma}-1$ ), where $s$ and $t$ are odd integers bigger than $1 ; \alpha, \beta$ and $\sigma$ are positive integers that with $\alpha \geq 1, \beta>0$ and $\sigma>0$; then among $s^{\beta}$ consecutive odd numbers next to $a$ 's left there are odd numbers $b_{i s}$ such that $s^{i s} \mid b_{i s}$, among $t^{\sigma}$ consecutive odd 
numbers next to $a$ 's left there are odd numbers $b_{i \sigma}$ such that $t^{i \sigma} \mid b_{i \sigma}$ where is $=\beta, \beta-1, \ldots, 2,1 \quad$ and $i \sigma=\sigma, \sigma-1, \ldots, 2,1$; among $s^{\beta}$ consecutive odd numbers next to $a$ 's right there are odd numbers $c_{i s}$ such that $s^{i s} \mid c_{i s}$ and among $t^{\sigma}$ consecutive odd numbers next to $a$ 's right there are odd numbers $c_{i \sigma}$ such that $t^{i \sigma} \mid c_{i \sigma}$ where $i s=\beta, \beta-1, \ldots, 2,1$ and $i \sigma=\sigma, \sigma-1, \ldots, 2,1$.

Proof. (Omitted)

Theorem 7. Let $s>1$ be an odd number and $\beta>1$ be a positive integer; suppose $e_{\beta}$ is an odd number such that $s^{\beta} \mid e_{\beta}$; then $s^{\alpha} \mid\left(e_{\beta} \pm 2 \lambda_{\omega} s^{\alpha}\right)$, where $\alpha$ and $\lambda_{\omega}$ are positive integers with $1 \leq \alpha \leq \beta$, and $\lambda_{\omega} \geq 1$.

Proof. $s^{\beta} \mid e_{\beta}$ yields $e_{\beta}=s^{\beta} t$ with some odd integer $t>1$; then $e_{\beta} \pm 2 \lambda_{\omega} s^{\alpha}=s^{\beta} t \pm 2 \lambda_{\omega} s^{\alpha}=s^{\alpha}\left(s^{\beta-\alpha} t \pm 2 \lambda_{\omega}\right)$.

Theorem 8. Let $p>1$ be an odd number and $\beta>1$ be a positive integer; then there are $p^{\beta-1}-1 p$ 's multiples in odd interval $\left(p^{\beta} e, p^{\beta}(e+2)\right)$, where $e$ is an odd integer.

Proof. Since $p$ and $e$ are odd, $e p^{\beta}, e p^{\beta}+2 p, e p^{\beta}+4 p, \ldots, e p^{\beta}+2 k p, \ldots, e p^{\beta}+2 p^{\beta}$ are all $p$ 's multiples. Eliminating the starting number $p^{\beta} e$ and the ending number $p^{\beta}(e+2)$, which are multiples of $p^{\beta}$, remains $p^{\beta-1}-1 p^{\text {'s multiples }}$ that are given by

$$
e p^{\beta}+2 p, e p^{\beta}+4 p, \ldots, e p^{\beta}+2 k p, \ldots, e p^{\beta}+2 p^{\beta}-4 p, e p^{\beta}+2\left(p^{\beta-1}-1\right) p
$$

Theorem 9. Suppose $p>1, e_{0}>1$ are odd numbers that satisfy $p \mid e_{0}$ and let $e=\left|\frac{e_{0}}{p^{2}}\right\rangle ;$ then $e_{0} \in\left[p^{2} e, p^{2}(e+2)\right)$.

Proof. Let $\tilde{e}=\left\lfloor\frac{e_{0}}{p^{2}}\right\rfloor$; if $\tilde{e}$ is odd, then taking $e=\left\lfloor\frac{e_{0}}{p^{2}}\right\rfloor$ yields

$$
p^{2} e=p^{2}\left\lfloor\frac{e_{0}}{p^{2}}\right\rfloor \leq p^{2} \cdot \frac{e_{0}}{p^{2}}=e_{0}
$$

and

$$
p^{2}(e+2)=p^{2}\left(\left\lfloor\frac{e_{0}}{p^{2}}\right\rfloor+2\right)>p^{2} \cdot \frac{e_{0}}{p^{2}}=e_{0}
$$

If $\tilde{e}$ is even, then taking $e=\left\lfloor\frac{e_{0}}{p^{2}}\right\rfloor-1$ leads to

$$
p^{2} e=p^{2}\left(\left\lfloor\frac{e_{0}}{p^{2}}\right\rfloor-1\right)<p^{2} \cdot \frac{e_{0}}{p^{2}}=e_{0}
$$

and

$$
p^{2}(e+2)=p^{2}\left(\left\lfloor\frac{e_{0}}{p^{2}}\right\rfloor+1\right)>p^{2} \cdot \frac{e_{0}}{p^{2}}=e_{0}
$$


Theorem 10. Suppose odd numbers $e_{0}$ and $p$ satisfy $e_{0}>p>1$; let $\beta>1$ be a positive integer and $e=\left|\frac{e_{0}}{p}\right\rangle$, then $\left[p^{\beta} e_{0}, p^{\beta}\left(e_{0}+2\right)\right] \subset\left[p^{\beta+1} e, p^{\beta+1}(e+2)\right]$.

Proof. Let $\tilde{e}=\left\lfloor\frac{e_{0}}{p}\right\rfloor$. Direct computation can show that $e=\tilde{e}=\left\lfloor\frac{e_{0}}{p}\right\rfloor$ matches to the theorem if $\tilde{e}$ is odd, and $e=\tilde{e}-1=\left\lfloor\frac{e_{0}}{p}\right\rfloor-1$ matches to the theorem if $\tilde{e}$ is even.

In fact, when $\tilde{e}$ is odd, both $p^{\beta+1} e$ and $p^{\beta+1}(e+2)$ are odd.

Since

and

$$
p^{\beta+1} e=p^{\beta+1}\left(\left\lfloor\frac{e_{0}}{p}\right\rfloor\right) \leq p^{\beta+1} \cdot \frac{e_{0}}{p}=p^{\beta} e_{0}
$$

$$
p^{\beta+1}(e+2)=p^{\beta+1}\left(\left\lfloor\frac{e_{0}}{p}\right\rfloor+2\right)>p^{\beta+1} \cdot\left(\frac{e_{0}}{p}+1\right)=p^{\beta}\left(e_{0}+2\right)
$$

it is sure that $\left[p^{\beta} e_{0}, p^{\beta}\left(e_{0}+2\right)\right] \subset\left[p^{\beta+1} e, p^{\beta+1}(e+2)\right]$.

If $\tilde{e}$ is even, then $e=\tilde{e}-1=\left\lfloor\frac{e_{0}}{p}\right\rfloor-1, p^{\beta+1} e$ and $p^{\beta+1}(e+2)$ are odd.

Since $\left\lfloor\frac{e_{0}}{p}\right\rfloor-1<\frac{e_{0}}{p}$, it yields

$$
p^{\beta+1} e=p^{\beta+1}\left(\left\lfloor\frac{e_{0}}{p}\right\rfloor-1\right)<p^{\beta+1} \cdot \frac{e_{0}}{p}=p^{\beta} e_{0},
$$

Next it shows $p^{\beta+1}(e+2) \geq p^{\beta}\left(e_{0}+2\right)$.

Actually,

$$
\begin{aligned}
& p^{\beta+1}\left(e_{2}+2\right)-p^{\beta}\left(e_{0}+2\right)=p^{\beta}\left(p e_{2}-e_{0}\right)+p^{\beta}(2 p-2) \\
& =p^{\beta}\left(p\left\lfloor\frac{e_{0}}{p}\right\rfloor+p-e_{0}\right)-2 p^{\beta}==p^{\beta}\left(p-\left(e_{0}-p\left\lfloor\frac{e_{0}}{p}\right\rfloor\right)\right)-2 p^{\beta} \\
& =p^{\beta}\left(p-\left(e_{0}-p\left\lfloor\frac{e_{0}}{p}\right\rfloor\right)\right)-2 p^{\beta}
\end{aligned}
$$

Note that $e_{0}-p\left\lfloor\frac{e_{0}}{p}\right\rfloor$ is the remainder of $e_{0}$ divided by $p$ and its value must be one of $1,3, \ldots, p-2$, the biggest of which is $p-2$; hence it holds $p-\left(e_{0}-p\left\lfloor\frac{e_{0}}{p}\right\rfloor\right) \geq 2$ and further

$$
p^{\beta+1}\left(e_{2}+2\right)-p^{\beta}\left(e_{0}+2\right)=p^{\beta}\left(p-\left(e_{0}-p\left\lfloor\frac{e_{0}}{p}\right\rfloor\right)\right)-2 p^{\beta} \geq 0
$$

$\square$

Example 3. Taking $p=3, e_{0}=5$ yields 


$$
\begin{aligned}
& {\left[3^{2} \times 5,3^{2} \times 7\right]=[45,63],\left\lfloor\frac{e_{0}}{p}\right\rfloor=1} \\
& \Rightarrow\left[3^{3} \times 1,3^{3} \times 3\right]=[27,81] \supset[45,63]
\end{aligned}
$$

Taking $p=5, e_{0}=15$

$$
\begin{aligned}
& {\left[5^{2} \times 15,5^{2} \times 17\right]=[375,425],\left\lfloor\frac{e_{0}}{p}\right\rfloor=3} \\
& \Rightarrow\left[5^{3} \times 3,5^{3} \times 5\right]=[375,625] \supset[375,425]
\end{aligned}
$$

Taking $p=5, e_{0}=11$ yields

$$
\begin{aligned}
& {\left[5^{2} \times 11,5^{2} \times 13\right]=[275,325],\left\lfloor\frac{e_{0}}{p}\right\rfloor=2} \\
& \Rightarrow\left[5^{3} \times 1,5^{3} \times 3\right]=[125,375] \supset[275,325]
\end{aligned}
$$

Taking $p=3, e_{0}=7$ yields

$$
\begin{aligned}
& {\left[3^{2} \times 7,3^{2} \times 9\right]=[63,81],\left\lfloor\frac{e_{0}}{p}\right\rfloor=2} \\
& \Rightarrow\left[3^{3} \times 1,3^{3} \times 3\right]=[27,81] \supset[63,81]
\end{aligned}
$$

and taking $p=3, e_{0}=19$ yields

$$
\begin{aligned}
& {\left[3^{2} \times 19,3^{2} \times 21\right]=[171,189],\left\lfloor\frac{e_{0}}{p}\right\rfloor=6} \\
& \Rightarrow\left[3^{3} \times 5,3^{3} \times 7\right]=[135,189] \supset[171,189]
\end{aligned}
$$

Corollary 6 Let $a=p s$ be an odd composite integer, where $p$ and $s$ are odd numbers with $1<p<s$; if $p^{k}<a<p^{k+1}$, then

$$
p^{k} b_{k-1}<\ldots<p^{2} b_{1}<a=p s<p^{2} c_{1}<\ldots<p^{k} c_{k-1}
$$

where $b_{1}=\left|\frac{s}{p}\right\rangle b_{i+1}=\left|\frac{b_{i}}{p}\right\rangle, c_{i}=b_{i}+2, i=1,2, \ldots, k-1$.

In other words, some of the $p$ 's powers, $p^{k}, p^{k-1}, \ldots, p^{2}$, are in some way symmetrically distributed as divisors of odd numbers around $a$, as depicted in figure 2 .

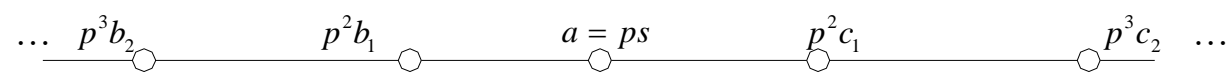

Figure 2. Symmetrically distributed powers of $a$ 's divisor $p$

Example 4. Odd number $46189=11 \times 4199$ satisfies $11^{4}<46189<11^{5}$;

then $b_{1}=\left\lfloor\frac{4199}{11}\right\rfloor=381, b_{2}=\left\lfloor\frac{381}{11}\right\rfloor=33, b_{3}=\left\lfloor\frac{33}{11}\right\rfloor=3$ and $c_{1}=383, c_{2}=35, c_{3}=5$, which result in

$$
\underbrace{11^{4} \times 3}_{43923}<\underbrace{11^{3} \times 33}_{43923}<\underbrace{11^{2} \times 381}_{46101}<\underbrace{11 \times 4199}_{46189}<\underbrace{11^{2} \times 383}_{46343}<\underbrace{11^{3} \times 35}_{46585}<\underbrace{11^{4} \times 5}_{73205}
$$

Corollary 7 Let $a=p s$ be an odd composite integer, where $p$ and $s$ are odd numbers with $1<p<s$; then there always exist an odd sequences $1 \leq b_{k} \leq \ldots \leq b_{2} \leq b_{1}$ and $c_{1} \geq c_{2} \geq \ldots \geq c_{k} \geq 1$ that satisfy

$$
p^{k+1} b_{k}<\ldots<p^{2} b_{1}<a=p s<p^{2} c_{1}<\ldots<p^{k+1} c_{k}
$$




\section{Conclusions}

Consecutive odd integers always express a sequence of odd numbers. Knowing the properties of consecutive odd integers is undoubtedly helpful for knowing the distribution of a odd integer's divisors, as stated in the theorems and corollaries that are proved in previous sections. This can also help people to design fast algorithms to find a divisor of an odd integer, and thus solve the problem of factoring big integers. This is my original intention and I hope the day come soon.

\section{Acknowledgments}

The research work is supported by Department of Guangdong Science and Technology under projects 2015A030401105 and 2015A010104011, Foshan Bureau of Science and Technology under projects 2016AG100311, and projects 2014SFKC30 and 2014QTLXXM42 from Guangdong Education Department. The authors sincerely present thanks to them all.

\section{References}

Dickson L. E. (1971). History of the Theory of Numbers, Chelesea publishing Company, New York.

Kessler, G. C. (2016). An Overview of Cryptography, ERAU Scholarly Commons.

Liu, X. X, Zou, X. X, \& Tan, J. L. (2014). Survey of large integer factorization algorithms, Application Research of Computers, 13(11), 3201-3207.

Rosen K. H. (2011). Elementary Number Theory and Its Applications(6th ed.). Addison-Wesley.

Sarnaik, S., Gadekar, D., Gaikwad, U. (2014). An Overview to Integer Factorization and RSA in Cryptography, International Journal for Advance Research in Engineering and Technology,2(IX),21-27

Wang Xingbo. (2014). New Constructive Approach to Solve Problems of Integers' Divisibility, Asian Journal of Fuzzy and Applied Mathematics, 2(3), 74-82.

Wang Xingbo. (2016). Seed and Sieve of Odd Composite Numbers with Applications in Factoriization of Integers. IOSR Journal of Mathematics, 12(5), 01-07.

Wang Xingbo. (2016). Valuated Binary Tree: A New Approach in Study of Integers, International Journal of Scientific and Innovative Mathematical Research, 4(3), 63-67.

Xingbo Wang. (2016).Amusing Properties of Odd Numbers Derived From Valuated Binary Tree, IOSR Journal of Mathematics, 12(6,Ver.V), 53-57.

Xingbo Wang. (2017). Genetic Traits of Odd Numbers With Applications in Factorization of Integers. Global Journal of Pure and Applied Mathematics, 13(1), 318-333.

Xingbo Wang. (2017). Two More Symmetric Properties of Odd Numbers. IOSR Journal of Mathematics, 13(3 Ver. II), 37-40. https://doi.org/10.9790/5728-1303023740

\section{Copyrights}

Copyright for this article is retained by the author(s), with first publication rights granted to the journal.

This is an open-access article distributed under the terms and conditions of the Creative Commons Attribution license (http://creativecommons.org/licenses/by/4.0/). 